\title{
Wideband Hybrid Precoding Techniques for THz Massive MIMO in 6G Indoor Network Deployment
}

\section{Jeyakumar P ( $\nabla$ jeyakumarpsg@gmail.com )}

National Institute of Technology Tiruchirappalli https://orcid.org/0000-0002-2043-790X

\section{Vishnu V}

National Institute of Technology Tiruchirappalli

\section{Srinitha S}

Sri Ramakrishna Engineering College

\section{Muthuchuchidambaranathan P}

National Institute of Technology Tiruchirappalli

\section{Arvind Ramesh}

National Institute of Technology Tiruchirappalli

\section{Research Article}

Keywords: Terahertz, Delay-phase Precoding, Beam Spilt effect, 6G Indoor Office, Channel Simulation

Posted Date: July 9th, 2021

DOI: https://doi.org/10.21203/rs.3.rs-568831/v1

License: (c) (1) This work is licensed under a Creative Commons Attribution 4.0 International License. Read Full License 


\title{
Wideband Hybrid Precoding Techniques for $\mathrm{THz}$ Massive MIMO in 6G Indoor Network Deployment
}

\author{
Jeyakumar $\mathbf{P}^{1}$, Vishnu $\mathbf{V}^{2}$, Srinitha $\mathbf{S}^{2}$, \\ Muthuchidambaranathan $\mathbf{P}^{1}$,Arvind \\ Ramesh $^{1}$
}

Received: date / Accepted: date

\begin{abstract}
Terahertz (THz) communication is becoming an up-and-coming technology for the future $6 \mathrm{G}$ networks as it provides an ultra-wide bandwidth. Appropriate channel models and precoding techniques are essential for supporting the desired coverage and mainly to resolve the severe path loss in $\mathrm{THz}$ signals. Initially, the Sub- $\mathrm{THz}$ channel $(140 \mathrm{GHz})$ impulse response by using NYUSIM Channel Simulator for $6 \mathrm{G}$ indoor office scenario is investigated in this work. The highlight is on Large scale and Small scale parameters like propagation delay and path loss, antenna array gain, etc. The beam split effect is a critical challenge of $\mathrm{THz}$ wideband communication. Therefore We have proposed three different $\mathrm{THz}$ precoding methodologies like the hybrid precoding, analog beamforming, and the delay-phase precoding to address this challenge.
\end{abstract}

Jeyakumar $\mathrm{P}^{1}$

Department of Electronics and Communication Engineering,

National Institute of Technology, Tiruchirappalli 620015,India

E-mail: jeyakumarpsg@gmail.com

Vishnu $\mathrm{V}^{2}$

Department of Electronics and Communication Engineering,

National Institute of Technology, Tiruchirappalli 620015,India

E-mail: vishnuv0209@gmail.com

Srinitha $\mathrm{S}^{2}$

Department of Electronics and Communication Engineering,

Sri Ramakrishna College of Engineering, Coimbatore, India

E-mail: srinitha@srec.ac.in

Muthuchidambaranathan $\mathrm{P}^{1}$

Department of Electronics and Communication Engineering, National Institute of Technology, Tiruchirappalli 620015,India

E-mail: muthuc@nitt.edu

Arvind Ramesh ${ }^{1}$

Department of Instrumentation and Control Engineering,

National Institute of Technology, Tiruchirappalli 620015,India

E-mail: arvindramesh25@gmail.com 
We then extensively investigate its diverse number of time delayers, varying number of antenna elements, and comparison with frequency - mmWave and Sub-THz have been discussed. Finally, the proposed delay-phase precoding techniques outperforms the other precoding techniques with $97 \%$ of optimal precoding. So, this an efficient approach for implementing the future indoor communication network deployment for $6 \mathrm{G}$.

Keywords Terahertz, Delay-phase Precoding, Beam Spilt effect, 6G Indoor Office, Channel Simulation

\section{Introduction}

The International Tele-communication Union(ITU) launched the official research investigation over $6 \mathrm{G}$ helps to design pioneer wireless networks and also to attain self-subsisting networks. To quench out the emerging services and the applications like augmented reality, holographic communications, extremely high definition transmission of videos, the Tera-Hertz(THz) communications acts as a backbone for the future $6 \mathrm{G}$ wireless networks. $6 \mathrm{G}$ also provides the communication with reduced latency for long distance with ultra high reliability. The $\mathrm{THz}$ band ranges from $0.1 \mathrm{THz}$ to $10 \mathrm{THz}$ provides significant bandwidth owing to attain ultra-high data rate. Several interpretations over $6 \mathrm{G}$ be has a belief that $6 \mathrm{G}$ provides an empowered full-dimensional coverage with unlimited wireless connectivity.

For wireless communications, the peak data rate is considered to be an essential indicator to measure its effectiveness and in order to accomplish the above visions of $6 \mathrm{G}$, the peak data rate should be greater than 1 Tbps [1], [2]. Nevertheless, this peak data rate will not be supported by the existing $5 \mathrm{G}$ millimeter wave (mmWave) bandwidth. Comparing THz band, which is in 0.1 $\mathrm{THz}-10 \mathrm{THz}$ range with the mmWave, the $\mathrm{THz}$ band provides the significant bandwidth, for example, the bandwidth greater than $20 \mathrm{GHz}$ is provided to accomplish the extremely high data rate. Therefore, it is extensively believed that the communication through $\mathrm{THz}$ band is significant technology for the emerging 6G wireless networks [2]. The bandwidth provided should be used effectively in multi-user networks. An autonomous and large dimensional networks are the key features of $6 \mathrm{G}$ to provide wide coverage and ubiquitous connectivity.

The bandwidth obtained in the sub $6 \mathrm{GHz}$ of band, and the mmWave band is insubstantial to gratify the exigencies of the users in sixth generation era. The usage of available spectrum is started moving towards the $\mathrm{THz}$ band in bandwidth hungry applications [3]. In the applications of cellular, biological, molecular and vehicular communications, distinct use cases are encountered [4-6]. Owing to the bottleneck of large path loss, the THz communication is circumscribed to employ in short range applications. A good number of advanced applications like personal area networks, $6 \mathrm{G}$ communication, chip to chip communication uses $\mathrm{THz}$ band communication [7]. 
But the $\mathrm{THz}$ signals are often affected by the path loss for example, at 0.6 $\mathrm{THz}$ the path loss of $120 \mathrm{~dB} / 100 \mathrm{~m}$ will occur. Due to this path loss issue it becomes challenging to accomplish the expected coverage. The precoding approach helps to resolve path loss problem and in this approach there is no need to increase the power at the transmitter. By using this precoding methodology, narrow beams can be generated with large antenna array gain that combats the severe path loss and also the entire optimization process will be simplified into sub rate optimization processes and its complexity is evaluated [8].

The scale of the antenna array is directly proportional to the array gain of the emerging beam. The wavelength of the signal obtained from the $\mathrm{THz}$ band is considerably very small, therefore in $\mathrm{THz}$ communication, antenna arrays of very large scale is employed. As the optimization of path loss of $\mathrm{THz}$ signals can be used by $\mathrm{THz}$ precoding, which is a requisite methodology for $6 \mathrm{G}$ wireless networks. When compared to the precoding approach used in $5 \mathrm{G}$ mmWave systems, the $6 \mathrm{G}$ precoding techniques is facing new challenges because of its varied characteristics and these challenges should detected and resolved by an effective $6 \mathrm{G}$ system.

The major bottleneck of current wireless communication system is the limitation of available spectrum and because of that the significant quality of service cannot be provided and this can be alleviated by adopting the $\mathrm{THz}$ communication. The spectrum gridlock can be removed by applying novel methodologies and new frequency bands. For effective $\mathrm{THz}$ communication, the array-of-sub-array architecture is compared with the fully connected architecture. The comparison is made in terms of energy efficiency, spectral efficiency, power consumption, channel estimation etc., Highly complex and power consuming hardware is required for $\mathrm{THz}$ communication and many new communication strategies are applied owing to the nature of hardware [9]. In order to realize a wireless backhaul with an ultra-high speed, it is vital to analyze the bandwidth, transmission distance and the physical properties of the channel. To address these challenges in $\mathrm{THz}$ band, the distance can be enhanced by using distance aware bandwidth adaptive methodology. This approach capture all the distinct eccentricities of the channel and uses a full spectrum of resources by enabling several high-speed links. Highly advanced communication methodologies are needed to enhance the distance for transmission and this helps to provide simultaneous operation of ultra high speed links [10].

\section{Related work}

In 6G networks, an ultra broadband connectivity is offered by mounting the $\mathrm{THz}$ access points over the lamp post in the street which helps to provide the better bandwidth availability at the $\mathrm{THz}$ frequency range and this also becomes feasible for offloading the traffic from the access point (AP). As high frequencies are bounded by the smallest wavelength, the MIMO configuration 
is realized as the user equipment and the access points are outfitted with the arrays that constitutes large number of antenna elements. By using the pertinent beamforming methodology, the pitfall of having higher path loss is overcome by the substantial array gain and the interference caused by multiple users is facilitated by boosting the capacity of the system and to provide beams with high directivity in the massive MIMO configuration [11],[12].

$6 \mathrm{G}$ networks provides appreciable user throughput but it is also necessary to gratify the system requirements to improve the energy and the spectrum efficiency [13]. These network communication system is also anticipated to perform its operation with cost reduction and limited energy consumption [14]. Depending on the intent of the application, the performance trade offs regarding cost, efficiency and the computation complexity influences the appropriate precoding methodology [15]. Two stages of hybrid precoder constitutes analog beamformer and the digital precoder and these hybrid precoders uses only limited RF chains.

In the traditional hybrid precoding approach, full array gain is achieved by aligning the narrow beam from the analog beamformer in the direction of the intended users [16]. Nevertheless, in 5G mmWave massive MIMO systems, the generated beams from various sub-carrier frequencies concentrate on various physical directions because of the usage of phase shifters which is independent of frequency and this leads to loss in array gain [16]. Various methods are utilized to tackle this array gain loss which is encountered by the beam squint effect [17]-[21]. An optimized closed form solution is proposed in orthogonal frequency division multiplexing to deal with the hybrid precoding problem in the wide band massive MIMO system[18]. To enhance the hybrid precoding approach performance, an optimal solution is proposed to optimize the digital precoder and an analog beamformer iteratively in order to attain the significant performance over the whole bandwidth [1].

Additionally, the design of code books which contains beams of wide bandwidth is made in order to mitigate the beam squint effect which causes loss in array gain [20],[21]. The design of wide beams having reduced array gain is attained in each sub-carrier where a semi-definite relaxation methodology is used to enhance the overall antenna array gain over the whole bandwidth [20],[21]. The suggested methods show effectiveness to enhance the rate performance because the beams are squinted slightly and in case of mmWave massive MIMO systems, the loss in array gain is not a major consideration [18]-[21]. But these methods are not highly effective for $\mathrm{THz}$ band massive MIMO communication systems. The generated beams obtained at various sub-carrier frequencies decompose into individual physical directions because of the substantial number of antennas and wide bandwidth of $\mathrm{THz}$ signal.

The decomposition of different subcarrier beams in different directions is called as beam split and this is the fundamental difference between $\mathrm{THz}$ and mmWave beamforming systems. The beam split effect gives each subcarrier in the signal a different direction, where the subcarriers around the center frequency diverge around boresight of the beam. It is inferred that the significant array gain will be attained only on the generated beams around the 
central frequency. And the remaining beams suffer from a high loss in array gain. Consequently, the achievable rate is degraded by beam split effect. This can be overcome by using delay-phase precoding in this work[22,23].

\subsection{Major Contributions}

- The key contribution of this work is to apply the channel model to evaluate the $6 \mathrm{G}$ THz Massive MIMO system performance with NYUSIM Channel Simulation to verify the channel model parameters and antenna properties.

- Large scale and Small scale parameters have been discussed for 6G indoor office scenario for $140 \mathrm{GHz}$ operating frequency under UMi LOS environment at $0 \mathrm{dBm}$ Transmit power with coverage of $100 \mathrm{~m}$.

- Three precoding techniques have been investigated to deploy 6G single cell multi-user indoor office scenarios. The beam split effect is one reason behind to propose the delay-phase precoding technique for reducing the array gain loss that occurs.

- Finally, by varying the number of time delayers, frequency (mmWave and Sub-THz), and the number of transmitting antennas in the proposed delayphase precoding technique, the performances have been compared.

The paper is structured as Section 3 explains network deployment model with the small scale fading, large scale fading, power delay profile analysis. Section 4 illustrates various precoding methods like hybrid precoding, analog beamforming, delay-phase precoding techniques, and performance evaluation. The design approach is explained under section 5. Section 6 demonstrates simulation results with its inferences. Finally, Section 7 concludes the article.

\section{Network deployment Model}

Fig. 1 depicts the massive MIMO THz network deployment model. In indoor office scenarios, the major focus will be in the downlink, where the single cell access point (AP) is connected to multiple users. The small scale and large scale fading channel models, the structure of an antenna array, and the power delay profile analysis are discussed in the following subsections using NYUSIM [24].

\subsection{Large Scale Fading}

With NYUSIM, the expression for Close In (CI) free-space reference distance path loss model having one meter of reference distance with an additional 
attenuation caused by diversified atmospheric conditions were applied [26][29], and the expression is given as:

$$
P L^{C I}(f, d)[d B]=F S P L(f, 1 m)[d B]+10 n \log _{10}(d)+A T[d B]+\chi_{\sigma}^{C I}
$$

, where $\mathrm{d} \geq 1 \mathrm{~m}$

$d$ represents the three dimensional(3D) receiver-transmitter separation distance, 'f' represents carrier frequency in $\mathrm{GHz}$, where 'n' denotes the path loss exponent and the attenuation term is denoted by AT which is induced by atmosphere, the path loss in free space $(\mathrm{dB})$ is denoted by $\operatorname{FSPL}(f, 1 \mathrm{~m})$ with one meter of separation between transmitter and receiver at $f$ and $\chi_{\sigma}^{C I}$ represents a Gaussian random variable with zero-mean and standard deviation in $\mathrm{dB}$ :

$$
F S P L(f, 1 m)[d B]=20 \log _{10}\left(\frac{4 \pi f \times 10^{9}}{c}\right)=20 \log _{10}(f)+32.4[d B]
$$

where $c$ represents the speed of light in a vacuum and $f$ is the frequency in GHz. The characterisation of AT is given as:

$$
A T[d B]=\alpha[d B / m] \times d[m]
$$

The attenuation factor $(\mathrm{dB} / \mathrm{m})$ is denoted by ' $\alpha$ ' at $1 \mathrm{GHz}$ to $100 \mathrm{GHz}$ of frequency, that constitutes the combined effects of attenuation of haze, rain, dry air and water vapor [27]. Here ' $d$ ' represents the 3D transmitter-receiver distance of separation in (1).

\subsection{Small-Scale Fading}

The double-directional channel impulse response (CIR) $h_{\text {dir }}$, in small scale fading, having ' $\mathrm{L}$ 'multi-path components for every transmission link will be provided as follows:

$$
h_{d i r}(t, \phi)=\Sigma_{l=1}^{L} P_{R X, l} \cdot e^{j \varphi_{l}} \cdot \delta\left(t-\tau_{l}\right) \cdot G_{T X}\left(\phi-\phi_{l}^{T X}\right) \cdot G_{R X}\left(\phi-\phi_{l}^{R X}\right)
$$

Here $G_{T X} \quad G_{R X}$ represents antenna gain at transmission and reception. $P_{R X, l}, \tau_{l}$ and $\phi_{l}$ denotes the magnitude of received power,propagation time delay and phase in the multi-path components. $\phi$ represents azimuth angle offset and ' $t$ ' denotes time. In every multi-path component, $\phi_{l}^{T X}$ denotes the angle of departure at the access point and $\phi_{l}^{R X}$ represents the angle of arrival for every mobile users (MUs). 


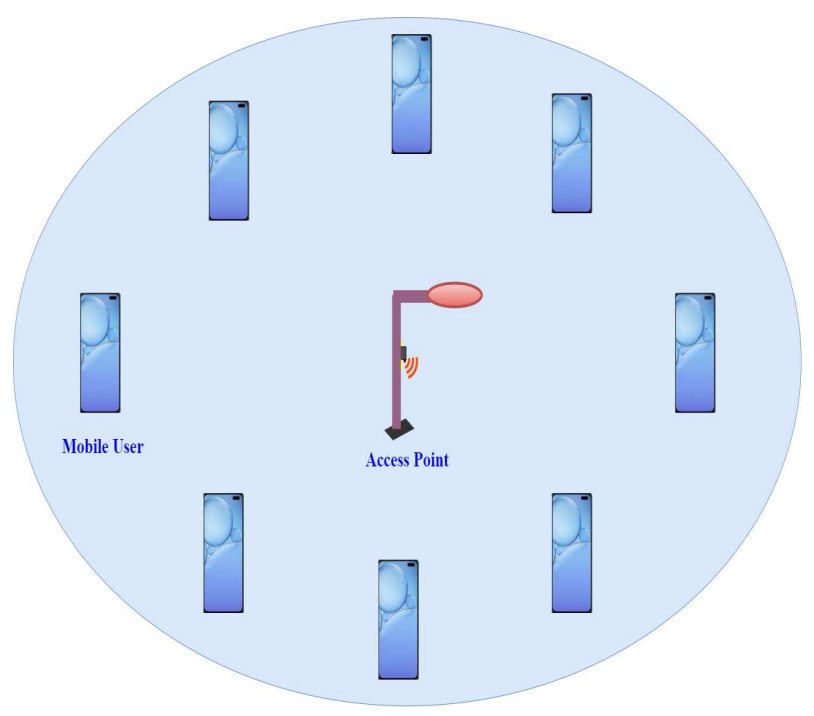

Fig. 1: Network deployment model

\subsection{Power Delay Profile Analysis}

To create a communication link between the APs and MUs and to maintain the desired data rate of a channel, the received power at the MUs should be modified accordingly. The study of the power delay profile is critical for network deployment. As shown in Table 1, there are a total of 32 input parameters are given as input to the simulator that is categorized into two divisions: antenna properties and channel parameters. The panel antenna properties are made up of 12 input parameters that are linked to the antenna arrays at transmission and reception, while the panel channel parameter is made up of 20 input parameters that provide information on the propagation channel. The proposed network parameters for urban microcell (UMi) indoor $\mathrm{THz}$ communication systems are described in Table 1 . The APs use a carrier frequency of 0.14 $\mathrm{THz}$ and transmit power of $0 \mathrm{dBm}[30]$. The NYUSIM statistical parameters at $0.14 \mathrm{THz}$ LOS channel path-loss model is given by free space path loss $P L_{0}$ $=255.32 \mathrm{~dB}$.

The power spectrum of 3D Angle of Departure (AOD) is shown in Figure $2(\mathrm{a})$, and the power spectrum of 3D Angle of Arrival(AOA) is shown in Figure 2(b) which have been simulated in NYUSIM. Whereas, the Figure 3(a) depicts the corresponding simulated omni-directional Power delay profile. For the 0.14 $\mathrm{THz}$ UMi LOS environment, the separation between transmitter and receiver is held at $100 \mathrm{~m}$. The received power is $-122.8 \mathrm{dBm}$ and the path delay $\sigma$ is $17.9 \mathrm{~ns}$ having a Path Loss Exponent (PLE) of 2.4. The directional power delay profile for a $0.14 \mathrm{THz}$ UMi LOS area with a PLE of 2.7 and transmitter and receiver antenna half power beamwidths (HPBW) of $8^{\circ}$ azimuth and $8^{\circ}$ elevation as shown in Figure 3(b). Both the transmitter and receiver antennas 
Table 1: Input parameters settings

\begin{tabular}{|c|c|}
\hline Parameters & Value \\
\hline Frequency $(\mathrm{GHz})$ & 140 \\
\hline RF bandwidth(MHz) & 1000 \\
\hline Scenario & UMi \\
\hline Environment & LOS \\
\hline Lower Bound of T-R Separation Distance $(\mathrm{m})$ & 100 \\
\hline Upper Bound of T-R Separation Distance $(\mathrm{m})$ & 100 \\
\hline TX Power $(\mathrm{dBm})$ & 0 \\
\hline Base Station Height $(\mathrm{m})$ & 35 \\
\hline User Terminal Height $(\mathrm{m})$ & 1.5 \\
\hline Number of Rx Locations & 16 \\
\hline Barometric Pressure (mbar) & 1013.25 \\
\hline Humidity(\%) & 50 \\
\hline Temperature $\left({ }^{\circ} \mathrm{C}\right)$ & 20 \\
\hline Rain Rate in $\mathrm{mm} / \mathrm{hr}$ & 0 \\
\hline Polarization & Co-Pol \\
\hline Foliage Loss & No \\
\hline Distance within Foliage $(\mathrm{m})$ & 0 \\
\hline Foliage Attenuation $(\mathrm{dB} / \mathrm{m})$ & 0.4 \\
\hline Outdoor to Indoor(O2I) Penetration Loss & No \\
\hline O2I Loss Type & Low Loss \\
\hline TX Array Type & ULA \\
\hline RX Array Type & ULA \\
\hline Number of TX Antenna Elements $\left(N_{t}\right)$ & 256 \\
\hline Number of RX Antenna Elements $\left(N_{r}\right)$ & 1 \\
\hline TX Antenna Spacing(in wavelength) & 0.5 \\
\hline RX Antenna Spacing(in wavelength) & 0.5 \\
\hline Number of TX Antenna Elements Per Row $W_{t}$ & 1 \\
\hline Number of RX Antenna Elements Per Row $W_{r}$ & 1 \\
\hline TX Antenna Azimuth HPBW & $8^{\circ}$ \\
\hline TX Antenna Elevation HPBW & $8^{\circ}$ \\
\hline RX Antenna Azimuth HPBW & $8^{\circ}$ \\
\hline RX Antenna Elevation HPBW & $8^{\circ}$ \\
\hline
\end{tabular}

have a gain of $26.5 \mathrm{dBi}$ and the received power is $-76.4 \mathrm{dBm}$ with path delay, $\sigma=0.9$ ns. Figure 4(a) depicts a small scale PDP for indoor deployments with a Tx-Rx separation distance of $100 \mathrm{~m}$ and a frequency of $0.14 \mathrm{THz}$. For the Tx and Rx gain of $26.5 \mathrm{dBi}$, the Path Loss for Omnidirectional, Directional and Directional-best for 0.14 Thz, UMi LOS is shown in Figure 4(b) with respect to Tx-Rx Separation distance.

Throughout this article, the following notations are used: A represents a matrix, 'a' denotes a vector and $a$ is a scalar. A(i) illustrates the $\mathrm{i}^{\text {th }}$ column of $\mathrm{A},(.)^{*}$ denotes conjugate transpose, $(.)^{T}$ denotes transpose and $\operatorname{tr}(\mathrm{A})$ is its trace. $\|\mathrm{A}\|$ is the Frobenius norm of $\mathrm{A}$, and $|A|$ is its determinant; [A $\mid \mathrm{B}]$ represents the horizontal concatenation; The $p$-norm of $a$ is represented as $\|a\|_{p}$; $\operatorname{diag}(\mathrm{A})$ is a vector generated by the diagonal elements of the matrix $\mathrm{A} ; I_{N}$ is the $N \times N$ identity matrix; $0_{M * N}$ is the $M \times N$ all-zeros matrix; $n \sim \mathcal{N}\left(\mu, \sigma^{2}\right)$ 


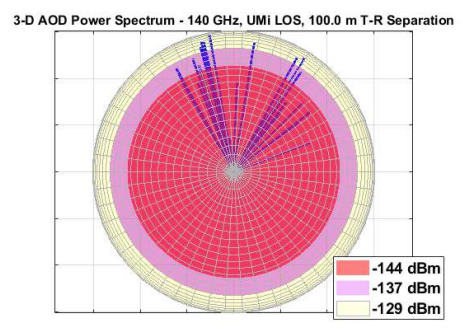

(a) 3D Power Spectrum of AoD

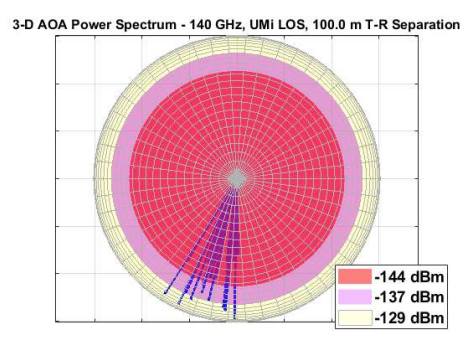

(b) 3D Power Spectrum of AoA

Fig. 2: Power Spectrum

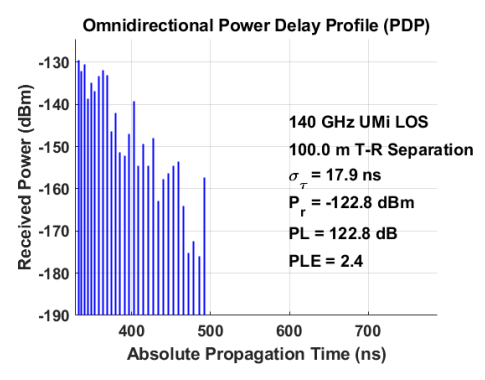

(a) Omnidirectional Power Delay Profile

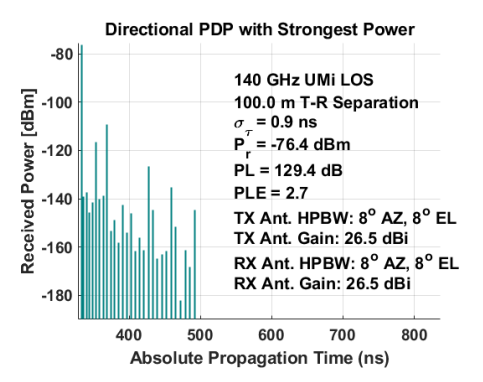

(b) Directional Power Delay Profile

Fig. 3: Power Delay Profiles

is the complex Gaussian vector of covariance $\sigma^{2}$ and mean 0. $E[$.] denotes the expectation and $R\{$.$\} denotes the real part of the variable.$

\section{Precoding Techniques}

For this precoding, we consider the multi-carrier system, by assuming an AP with $N_{t}$ antennas and $N_{R F}$ RF chains are communicated via ' $M$ ' sub carriers. The sampled transmitted signal is

$$
x[m]=F_{R F} F_{B B}[m] s[m], \text { for } m=1,2, \ldots, M,
$$

where s $=\left[s_{1}, s_{2}, \ldots, s_{M}\right]^{T}$ is the $M \times 1$ vector of transmitted symbols, such that $E\left[s[m] s^{*}[m]\right]=\frac{P_{t}}{M} I_{M}, F_{B B}=\left[f_{1}^{B B}, f_{2}^{B B}, \ldots, f_{M}^{B B}\right], F_{R F}=\left[f_{1}^{R F}, f_{2}^{R F}, \ldots, f_{M}^{R F}\right]$ 


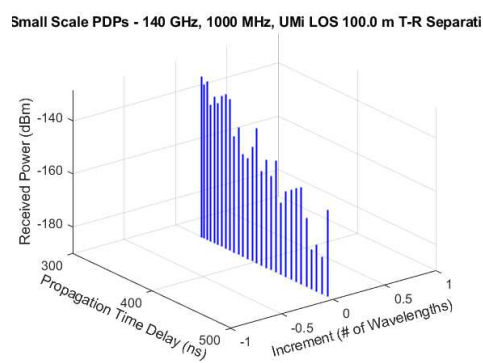

(b) Omnidirectional \& Directional Path Loss

(a) Small Scale Power Delay Profile

Fig. 4: Small scale PDPs \& Path Loss

and $P_{t}$ represents the average total transmitted power. In the time domain, the analog RF precoding $\left(F_{R F}\right)$ is performed and for the whole bandwidth, this same precoding matrix is employed. In the frequency domain, the digital baseband precoding $\left(F^{B B}[m]\right)$ is performed that is based on a per-subcarrier basis [18].

(i) Analog beamforming: Without the loss of generality, we assume the $l^{\text {th }}$ path component with physical direction of $\theta_{l}$ in (13). Generally, the $l^{\text {th }}$ column of the Analog Beamformer $F_{R F}$ in (7) and to obtain the analog beamforming vector $a_{l}$ :

$a_{l}=\frac{1}{N_{t}}\left[1, e^{-j 2 \pi \frac{d}{c} f_{c} \theta_{l}}, e^{-j 2 \pi \frac{2 d}{c} f_{c} \theta_{l}}, \ldots, e^{-j 2 \pi \frac{\left(N_{t}-1\right) d}{c} f_{c} \theta_{l}}\right]^{T}=f_{t}\left(2 \frac{d}{c} f_{c} \theta_{l}\right)=f_{t}\left(\theta_{l}\right)$

Where $N_{t}$ is number of transmitter antennas, $f_{c}$ is central frequency, c is speed of light in a vacuum, $d$ is the antenna spacing generally set according to $f_{c}$, the central frequency. The analog beamforming vector $a_{l}=F_{R F}[:, l]$, is used to generate the directional beam to the $l^{\text {th }}$ path's physical direction $\theta_{l}$, which is to be in near optimal [16]. Especially, the analog beamforming technique is to make electromagnetic waves which is transmitted by various antenna elements form an equal phase surface, which is perpendicular to the target physical direction $\theta_{l}[23]$.

(ii) Hybrid Precoding: In hybrid precoding, we introduce a baseband $\operatorname{precoder}\left(F_{B B}\right)$ in addition to the analog beamformer. The overall power constraint is imposed after normalizing $F_{B B}$ so that $\left\|F_{R F} F_{B B}\right\|_{F}^{2}=M$. A wide- 
band ray based channel model in (14) is considered, where the $m^{\text {th }}$ sub carrier the received signal $r_{m}$ as

$$
r_{m}=H_{m} \Sigma_{n=1}^{M} F_{R F} f_{n}^{B B} s_{n}+n_{m}
$$

where $H_{m}$ is the $N_{r} N_{t}$ matrix which denotes the channel present in between the $m^{\text {th }}$ sub carrier and the AP. The Gaussian noise with corrupting received signal is given by $n_{m} \sim N\left(0, \sigma^{2} I\right)$. For processing the received signal $y_{m}$, the $\mathrm{RF}$ combiner $w_{m}$ at the $m^{t h} \mathrm{MS}$ is given by:

$$
y_{m}=w_{m}^{*} H_{m} \Sigma_{n=1}^{M} F_{R F} f_{n}^{B B} s_{n}+w_{m}^{*} n_{m}
$$

where $w_{m}$ holds same constraints like RF precoders, that is the constant modulus and quantized angles constraints [18]. However, hybrid precoding is not ideal for capacity and flexibility requirements and can not be used in $\mathrm{THz}$ networks due to large array gain losses because of beam split effect [22].

(iii) Proposed Delay-Phase Precoding (DPP): Owing to the draw-

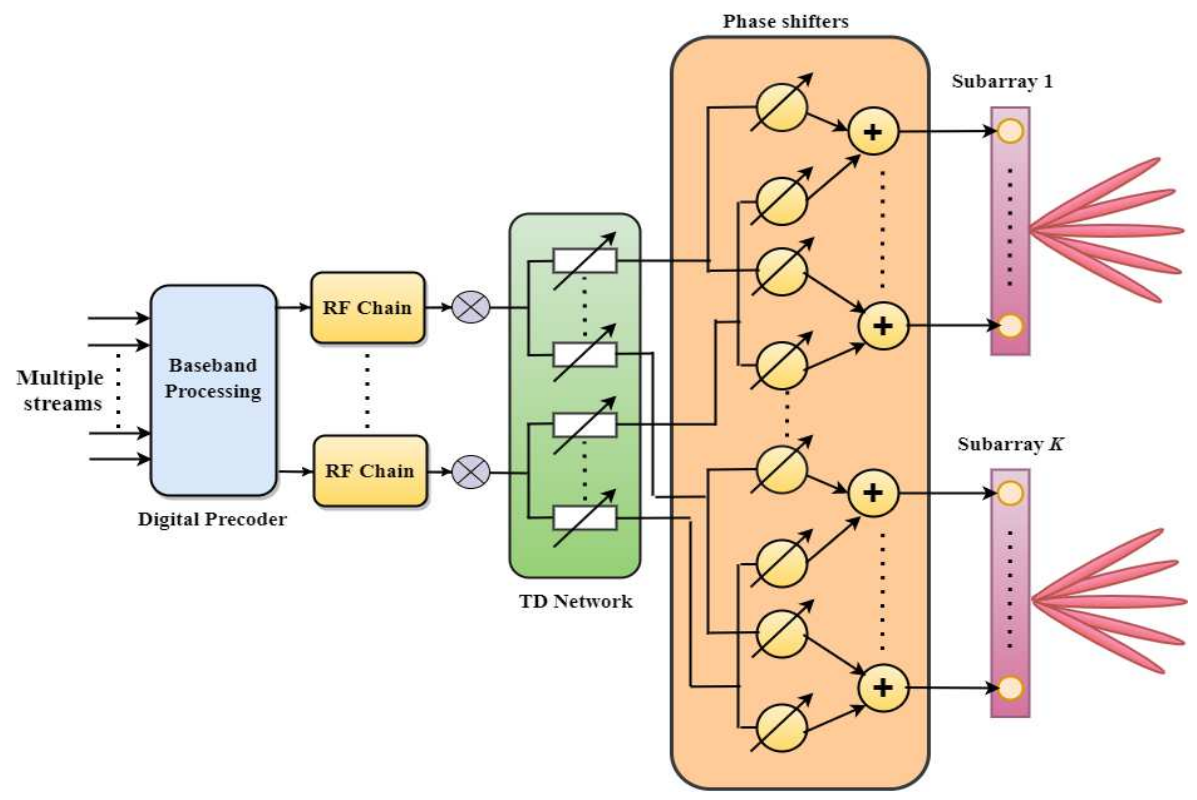

Fig. 5: The proposed DPP network

backs of the aforementioned precoding methods, a newfangled precoding methodology called Delay-phase precoding is proposed [22], [23]. In this technique we consider a conventional hybrid precoding with a $\mathrm{THz}$ massive MIMO system. The AP uses $N_{R F}$ radio-frequency chains and $N_{t}$-antenna uniform linear array(ULA). By serving an $N_{r}$-antenna user, the simultaneous transmission of $N_{s}$ data streams is done $\left(N_{s}=N_{r} \leq N_{R F} \ll N_{t}\right)$. The orthogonal frequency 
division multiplexing (OFDM) technique is adopted for realizing the reliable wide-band transmission having ' $\mathrm{M}$ ' sub-carriers. In between the conventional analog beamformer and the digital precoder, a time-delay(TD) network has been introduced. Here the suggested DPP helps to convert the conventional beamformer (phase controlled) into the controlled beamformer with combined delay-phase that is used in the realization of beamforming which is frequency dependent. Every RF chain is linked to ' $K$ ' TD elements and every TD element will be linked to ' $P_{t}=N_{t} / K$ ' conventional frequency independent phase shifters in a sub connected fashion. Therefore, at $m^{\text {th }}$ subcarrier, the received signal is represented as

$$
y_{m}=H_{m}^{H} \Sigma_{n=1}^{M} F_{R F_{n}} A_{m}^{T D} f_{m}^{B B} s_{m}+n_{m},
$$

where $H_{m} \in C^{N_{t} \times N_{r}}$ represents the $m^{t h}$ sub-carrier channel, the analog beamformer provided by the frequency-independent PSs is denoted as $F_{R F_{n}} \in$ $C^{N_{t} \times N_{R F}}$ with the form as

$$
F_{R F_{n}}=\left[F_{R F_{n, 1}}, F_{R F_{n, 2}}, \ldots, F_{R F_{n, N_{R F}}}\right]
$$

where $F_{R F_{n, l}}=\operatorname{blkdiag}\left(\left[\overline{a_{l, 1}}, \overline{a_{l, 2}}, \ldots \overline{a_{l, K}}\right]\right)$ represents the analog beamformer that is accomplished by the PSs linked to the $l^{\text {th }}$ RF chain through TDs, and $A_{m}^{T D} \in C^{K N_{R F} \times N_{R F}}$ represents the frequency-dependent phase shifts that is accomplished by TD network, that satisfies:

$$
A_{m}^{T D}=\operatorname{blkdiag}\left(\left[e^{-j 2 \pi f_{m} t_{1}}, e^{-j 2 \pi f_{m} t_{2}}, \ldots, e^{-j 2 \pi f_{m} t_{N_{R F}}}\right]\right)
$$

The baseband precoder is denoted by $F_{B B_{m}} \in C^{N_{R F} \times N_{s}}$ at the $m^{\text {th }}$ subcarrier and an additive white Gaussian noise (AWGN) is represented by $n_{u} \in$ $C^{N_{r \times 1}}$ at the $m^{t h}$ sub-carrier [22].

\subsection{Massive MIMO Channel Model}

A wide band 'ray-based' channel model is considered for the $\mathrm{THz}$ channel [22]. By denoting ' $f$ ' as bandwidth and ' $f_{c}$ ' as central frequency, the $m^{\text {th }}$ sub-carrier frequency is represented as follows:

$$
f_{m}=f_{c}+\frac{f}{M}\left(m-1-\frac{M-1}{2}\right), m=1,2, \ldots, M .
$$

For $m^{\text {th }}$ sub-carrier, the channel is given as

$$
H_{m}=\Sigma_{l=1}^{L} g_{l} e^{j 2 \pi \tau_{l} f_{m}} f_{t}\left(\theta_{l, m}\right) f_{r}\left(\phi_{l, m}\right)^{H}
$$

The total number of resolvable paths are represented by 'L', $\tau_{l}$ and $g_{l}$ denotes the path delay and path gain of the $l^{t h}$ path, $\theta_{l, m}, \phi_{l, m} \in[-1,1]$ represent the spatial direction of the transmitter and the receiver of the $l^{\text {th }}$ path and $m^{t h}$ sub-carrier, respectively, and $f_{t}\left(\theta_{l, m}\right), f_{r}\left(\phi_{l, m}\right)$ denotes the array responses in the transmitter and the receiver. 
For example, $f_{t}\left(\theta_{l, m}\right)$ is represented as follows:

$$
\begin{aligned}
f_{t}\left(\theta_{l, m}\right) & =\frac{1}{\sqrt{N_{t}}}\left[1, e^{j \pi \theta_{l, m}}, e^{j \pi \theta_{2 l, m}}, \ldots, e^{j \pi\left(N_{t}-1\right) \theta_{l, m}}\right]^{T} \\
f_{t}\left(\phi_{l, m}\right) & =\frac{1}{\sqrt{N_{r}}}\left[1, e^{j \pi \phi_{l, m}}, e^{j \pi \phi_{2 l, m}}, \ldots, e^{j \pi\left(N_{r}-1\right) \phi_{l, m}}\right]^{T}
\end{aligned}
$$

The direction of the paths in the spatial domain is the spatial direction that is determined by the subcarrier frequency and the physical propagation direction. For example, for the transmitter spatial direction, $\theta_{l, m}=2 d \frac{f_{m}}{c} \sin \gamma_{l}$, where $\gamma_{l} \in[-\pi / 2, \pi / 2]$ denotes the physical propagation direction of the $l^{t h}$ path, 'd' denotes the constant antenna spacing having $d=\frac{c}{2 f_{c}}$ and 'c' represents the speed of the light.

\section{Design Approach}

To accomplish three precoding techniques, an effective algorithm is proposed. The essence of this proposed algorithm lies in dividing the precoder calculation into three stages. To maximize the desired signal power, the analog beamformer is designed in stage- 1 and in the time delays are added to it in stage- 2 . In the third and last stage, the design of digital(baseband) precoder using the equivalent channel is done.

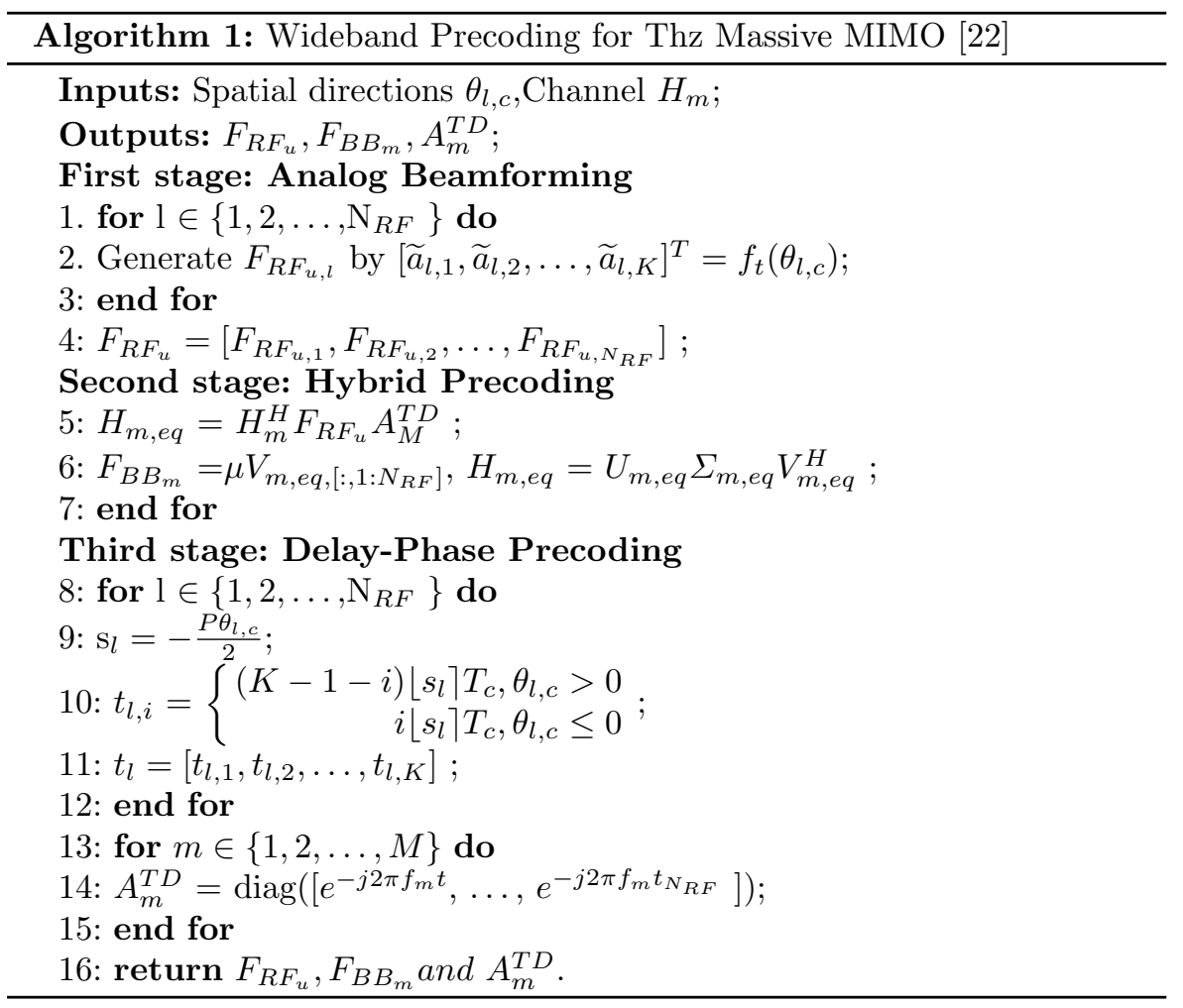


Table 2: Precoding Techniques

\begin{tabular}{|c|c|}
\hline Technique & Sumrate $(R)$ \\
\hline Analog Beamforming & $\frac{\frac{P_{t}}{M}\left|w_{m}^{H} H_{m} f_{m}^{R F}\right|^{2}}{\frac{P_{t}}{M} \Sigma_{n \neq m}\left|w_{m}^{H} H_{m} f_{n}^{R F}\right|^{2}+\sigma_{m}^{2}}$ \\
\hline Hybrid Precoding & $\frac{\frac{P_{t}}{M}\left|w_{m}^{H} H_{m} F_{R F} f_{m}^{B B}\right|^{2}}{\frac{P_{t}}{M} \Sigma_{n \neq m}\left|w_{m}^{H} H_{m} F_{R F} f_{n}^{B B}\right|^{2}+\sigma_{m}^{2}}$ \\
\hline Delay-Phase Precoding & $\frac{1}{M} \Sigma_{m=1}^{M} \log _{2}\left(\left|I_{N_{s}}+\frac{P_{t}}{N_{s} \sigma_{m}^{2}} H_{m} F_{R F_{m}} F_{B B_{m}} F_{B B_{m}}^{H} F_{R F_{m}}^{H} H_{m}^{H}\right|\right)$ \\
\hline
\end{tabular}

In this algorithm, the analog precoder for the $l^{\text {th }}$ beam $F_{R F_{u, l}}$ is estimated initially in the step 2 for generating the beams in the spatial direction $\theta_{l, c}$. After that the analog beamformer $F_{R F_{u}}$ is generated in step 4 . The time delays by ' $K$ ' TD elements have been generated in subsequent steps 6,7 and 8 , where the direction of beams are altered from $\theta_{l, c}$ to $\theta_{l, m}$ at the frequency $f_{m}$. Next in step 11, the analog beamformer with the time delay $A_{m}^{T D}$ is generated. Ultimately, in step 12 and 13, the digital precoder $F_{B B_{m}}$ is estimated depending upon the equivalent channel $H_{m, e q}$ by conventional singular value decomposition precoding method. From the Algorithm 1, the DPP achieves near-optimal achievable rate, as every beam is aligned with the spatial direction at all the sub-carriers by time delays. This will be further verified by using the results obtained from the simulation.

\subsection{Performance Evaluation}

The system's performance is calculated by using SE [(bits/s)/Hz] for every user. It is evaluated by (17) where the $S I N R_{m}$ for the considered precoding methods are tabulated under Table 2 . The system's sum rate $\mathrm{R}$ (bits/s) is given by (18)

$$
\begin{aligned}
& S E_{m}=\log _{2}\left(1+S I N R_{m}\right) \\
& R=\Sigma_{m=1}^{M}\left(B W_{m} \times S E_{m}\right)
\end{aligned}
$$

where $B W_{m}$ is the allocation of bandwidth for each user. Equal allocation of power is considered, where the total power is allocated uniformly for the users that is observed in the Table 2.

\section{Simulation results and discussion}

For comparing the achievable sum-rate of three different precoding methodologies along with the optimal fully-digital precoding, the simulation results are provided. A multi-user single cell $\mathrm{THz}$ system is considered $5 \mathrm{GHz}$ bandwidth at $0.14 \mathrm{THz}$ of carrier frequency. The AP equips a 256-element ULA for 
Table 3: Simulation Parameters for Precoding Techniques

\begin{tabular}{|c|c|}
\hline The number of the AP antennas $\mathrm{N}_{t}$ & 256 \\
\hline The number of the user antennas $\mathrm{N}_{r}$ & 1 \\
\hline Number of channel paths $\mathrm{L}$ & 4 \\
\hline The central frequency $f_{c}$ & $0.14 \mathrm{THz}$ \\
\hline The bandwidth B & $5 \mathrm{GHz}$ \\
\hline The number of the subcarriers M & 128 \\
\hline The number of RF chains $\mathrm{N}_{R F}$ & 4 \\
\hline The number of TD elements $\mathrm{K}$ & 4,16 \\
\hline Physical directions of the paths $\overline{\theta_{l}}, \overline{\phi_{l}}$ & $\mathcal{U}[\pi / 2, \pi / 2]$ \\
\hline The transmission SNR $\mathrm{P}_{t} / \sigma^{2}$ & $-20 \sim 15 d B$ \\
\hline
\end{tabular}

serving multiple users. The system parameters are shown in Table 3 . From the Fig $6 \&$ 7, the proposed Delay-phase precoding's achievable rate performance, Analog Beamforming and Hybrid precoding and is compared for the values of number of time delayers, $K=4$ and $K=16$ also it can be inferred that this DPP methodology outperforms other methodologies and attains $97 \%$ performance of the standard method, the optimal unconstrained full-digital precoding, due to the joint control of delay-phase.

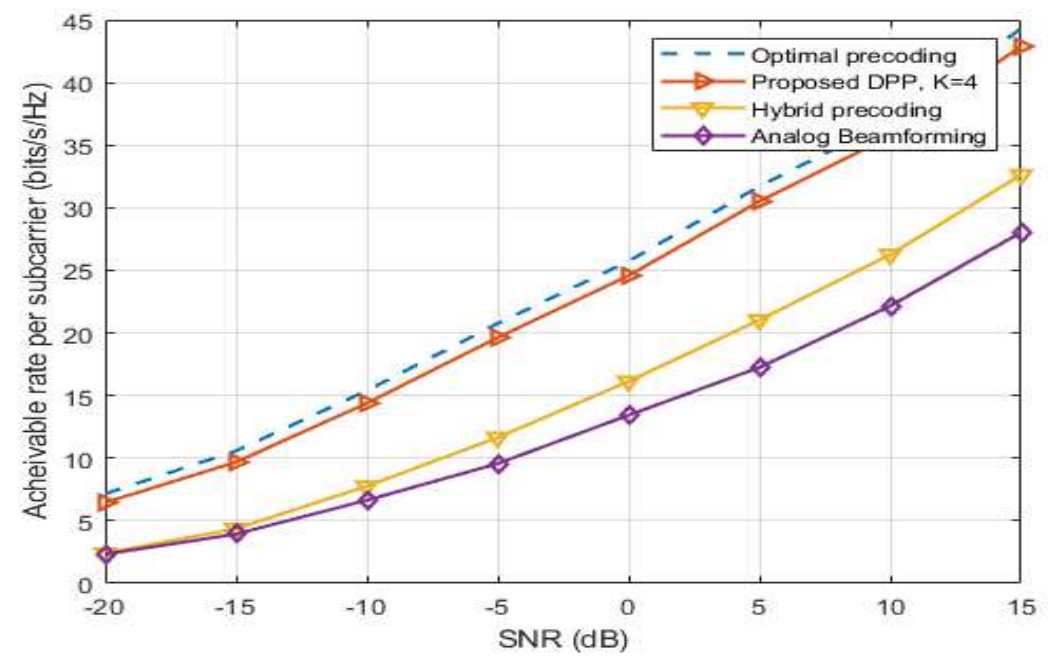

Fig. 6: Achievable rate performance comparison for 4 time delayers $(\mathrm{K}=4)$

Fig. 6 depicts the achievable sum-rate performance for different precoding techniques when the number of time delayers are set to 4. From the Fig. 6, the optimal fully-digital precoding's performance is been compared with the hybrid precoding structure with PSs, which is suffering from severe loss in the 


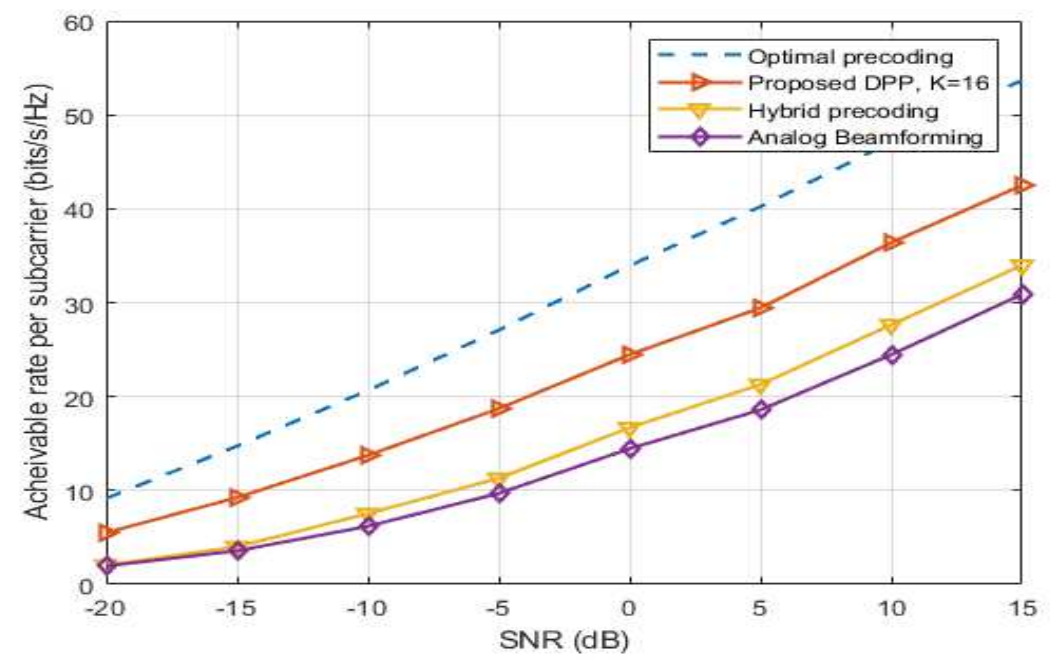

Fig. 7: Achievable rate performance comparison for 16 time delayers $(\mathrm{K}=16)$

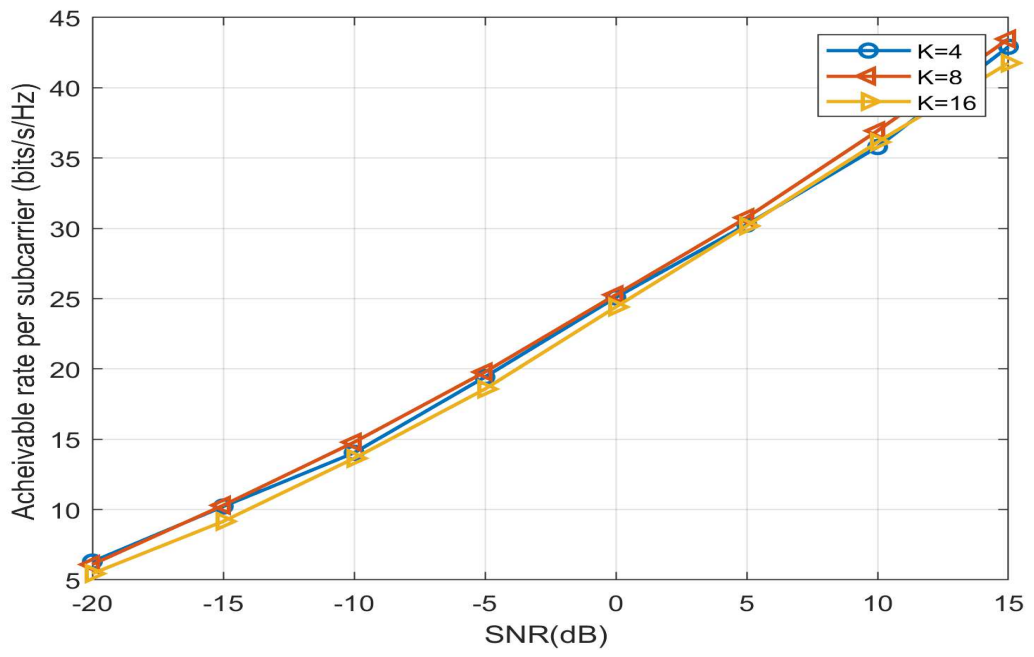

Fig. 8: The rate performance comparison for different number of time delayers

achievable sum-rate. It is due to the fact that they are not having capability to deal with the beam split effect. Conversely, hybrid precoding technique with TDs yield high performance. For the $\mathrm{K}=4$ the proposed DPP having achievable sum-rate of $42.86 \mathrm{bits} / \mathrm{s} / \mathrm{Hz}$ near to optimal precoding $(97 \%)$ which is $44.12 \mathrm{bits} / \mathrm{s} / \mathrm{Hz}$ from Table 4 . Similarly, the proposed DPP outperforms 


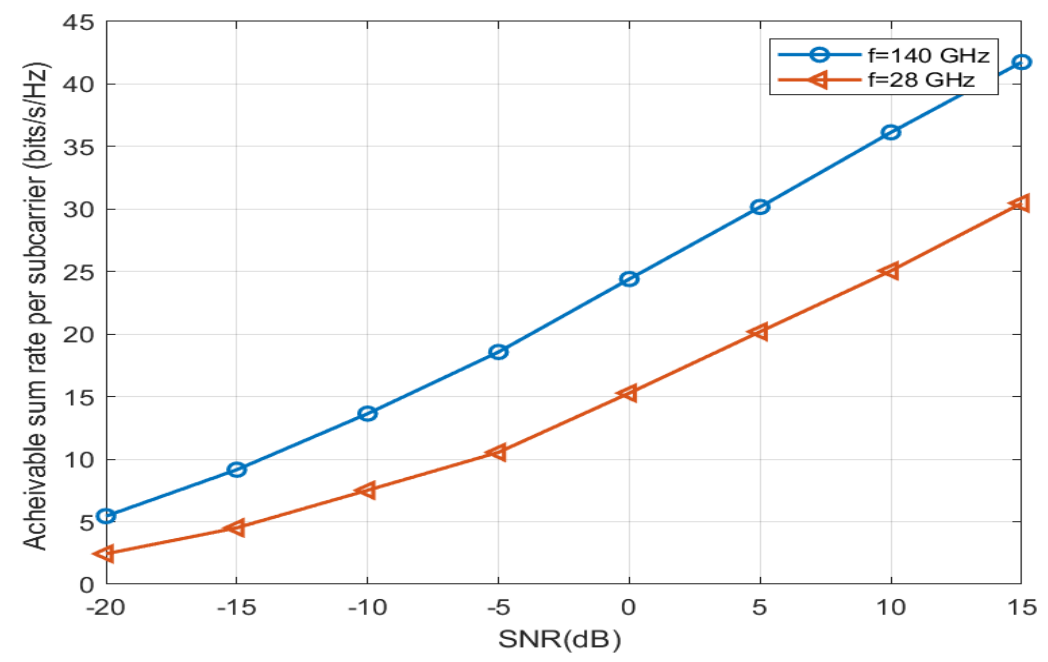

Fig. 9: The rate performance comparison for $\mathrm{THz}$ channel and mmWave channel

Table 4: Comparison of various precoding algorithms spectral efficiency $(\mathrm{K}=4)$ at $\mathrm{SNR}=15 \mathrm{~dB}$

\begin{tabular}{|c|c|c|}
\hline Precoding method & No. of Antenna & SE (bits/s/Hz) \\
\hline Optimal Precoding & 256 & 44.12 \\
\hline Analog Beamforming & 256 & 27.78 \\
\hline Hybrid Precoding & 256 & 32.65 \\
\hline Proposed DPP & 256 & 42.92 \\
\hline
\end{tabular}

Table 5: Comparison of various precoding algorithms spectral efficiency $(\mathrm{K}=16)$ at $\mathrm{SNR}=15 \mathrm{~dB}$

\begin{tabular}{|c|c|c|}
\hline Precoding method & No. of Antenna & SE (bits/s/Hz) \\
\hline Optimal Precoding & 256 & 53.7 \\
\hline Analog Beamforming & 256 & 30.89 \\
\hline Hybrid Precoding & 256 & 33.99 \\
\hline Proposed DPP & 256 & 41.76 \\
\hline
\end{tabular}

other methods like analog beamforming and hybrid precoding as per the Table 5 and Fig 7.

The proposed Delay-phase precoding network's performance with different number of time delayers has been compared in Fig 8. From the comparison, it is inferred that for the values of $K=4$ and $K=8$ are higher than the $K=16$. Therefore to implement the proposed network, the optimum value of the number of time delayers used in the circuit should be lesser, like $K=4$ or $K=8$ than choosing $\mathrm{K}=16$. 
Table 6: Comparison of various DPP precoding algorithms spectral efficiency for different time delayers at $\mathrm{SNR}=15 \mathrm{~dB}$

\begin{tabular}{|c|c|c|}
\hline Precoding method & No. of time delayers(K) & SE (bits/s/Hz) \\
\hline Proposed DPP & 4 & 42.92 \\
\hline Proposed DPP & 8 & 43.47 \\
\hline Proposed DPP & 16 & 41.76 \\
\hline
\end{tabular}

The proposed Delay-phase precoding network's performance in THz channel and in mmWave channel has been compared in Fig 9. It can be observed that its performance is much better at frequency, $\mathrm{f}=0.14 \mathrm{THz}$ (sub- $\mathrm{THz}$ channel) than at $\mathrm{f}=28 \mathrm{GHz}$ (mmWave channel). For THz channels, the DPP network is effectively being able to negate the beam split effect caused by the traditional phase-shifters and improve the performance rate substantially whereas in mmWave channel, the proposed DPP network is not able to effectively cancel out the beam squint effect.

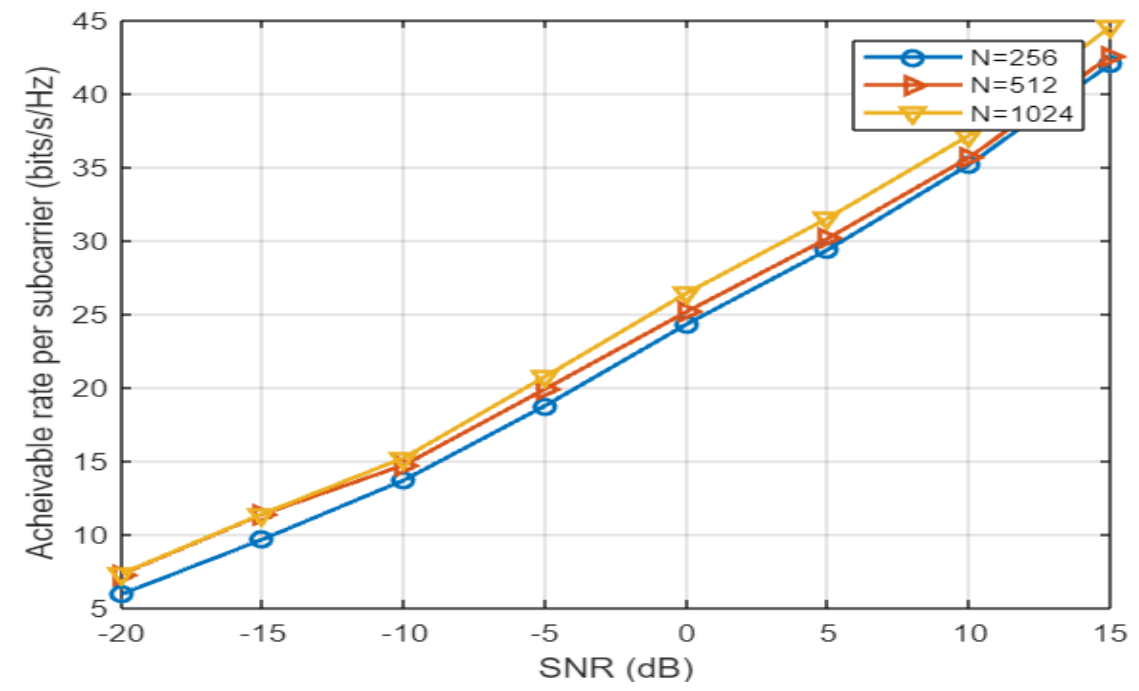

Fig. 10: Comparison of rate performance for different number of antennas

In the Fig 10, the performance of the proposed DPP is compared for various number of AP antennas $(\mathrm{N})$. There is not much difference observed when the value of $\mathrm{N}$ changes. Optimal performance is observed at $\mathrm{N}=1024$. Therefore, the DPP has the capability for solving the achievable rate degradation received by the beam split effect also to achieve more optimal achievable rate performance. 


\section{Conclusion}

This work verified the downlink single cell AP connected to multiple users for the $6 \mathrm{G}$ indoor office network deployment scenario and describes the channel model, the antenna properties, and power delay profile analysis employed using NYUSIM Channel Simulation. After that, we proposed a DPP technique where a TD network is being introduced to compensate the beam split effect and compared its performance to Hybrid precoding and Analog beamforming based on traditional frequency-independent phase shifters. From the simulation results and the theoretical analysis, it is observed that this DPP technique is capable to eradicate the beam split effect with more optimal (97\%) achievable rate performance. Under DPP Technique, the impacts of the carrier frequency of $28 \mathrm{GHz}$ mmWave and $140 \mathrm{GHz}$, the number of transmitting antennas and the number of time delayers on the achievable sum rate performance for a single cell multi-user scenario have been compared. This work will be further broadened for multi-cell scenario in future.

\section{Conflict of Interest:}

The authors declare that they have no conflict of interest

\section{References}

1. Z. Zhang, Y. Xiao, Z. Ma, M. Xiao, Z. Ding, X. Lei, G. K. Karagiannidis, and P. Fan, "6G wireless networks: Vision, requirements, architecture, and key technologies," IEEE Veh. Technol. Mag., vol. 14, no. 3, pp. 28-41, Sep. 2019. 2.T. S. Rappaport, Y. Xing, O. Kanhere, S. Ju, A. Madanayake, S. Mandal, A. Alkhateeb, and G. C. Trichopoulos, "Wireless communications and applications above $100 \mathrm{GHz}$ : Opportunities and challenges for 6G and beyond," IEEE Access, vol. 7, pp. 78 729-78 757, Jun. 2019.

3.Mumtaz, S., Jornet, J. M., Aulin, J., Gerstacker, W. H., Dong, X., Ai, B. (2017). Terahertz communication for vehicular networks. IEEE Transactions on Vehicular Technology, 66(7).

4. Akyildiz, I. F., Jornet, J. M., Han, C. (2014). Terahertz band: Next frontier for wireless communications. Physical Communication, 12, 16-32.

5. Lin, C., Li, G. Y. (2016). Energy-efficient design of indoor mmWave and sub-THz systems with antenna arrays. IEEE Transactions on Wireless Communications, 15(7), 4660-4672.

6. Busari, S. A., Mumtaz, S., Al-Rubaye, S., Rodriguez, J. (2018). 5G millimeterwave mobile broadband: Performance and challenges. IEEE Communications Magazine, 56(6), 137-143.

7. Busari, S. A., Khan, M. A., Huq, K. M. S., Mumtaz, S., Rodriguez, J. (2019). Millimetre-wave massive MIMO for cellular vehicle-to-infrastructure communication. IET Intelligent Transport Systems, 13(6), 983-990. 
8. X. Gao, L. Dai, S. Han, C. L. I, and R. W. Heath, "Energy-efficient hybrid analog and digital precoding for mmwave MIMO systems with large antenna arrays," IEEE J. Sel. Areas Commun., vol. 34, no. 4, pp. 998-1009, Apr. 2016. 9. C. Lin and G. Y. Li, "Terahertz communications: An array-of-subarrays solution," IEEE Commun. Mag., vol. 54, no. 12, pp. 124-131, Dec. 2016.

10. C. Han and I. F. Akyildiz, "Distance-aware bandwidth-adaptive resource allocation for wireless systems in the terahertz band," IEEE Trans. THz Sci. Technol., vol. 6, no. 4, pp. 541-553, Jun. 2016.

11.Busari, S. A., Huq, K. M. S., Mumtaz, S., Dai, L., Rodriguez, J. (2017). Millimeter-wave massive MIMO communication for future wireless systems: A survey. IEEE Communications Surveys Tutorials, 20(2), 836-869.

12.Alkhateeb, A., Leus, G., Heath, R. W. (2015). Limited feedback hybrid precoding for multi-user millimeter wave systems. IEEE transactions on wireless communications, 14(11), 6481-6494.

13.Chih-Lin, I. (2017). Seven fundamental rethinking for next-generation wireless communications. APSIPA Transactions on Signal and Information Processing, 6 .

14.Huq, K. M. S., Mumtaz, S., Bachmatiuk, J., Rodriguez, J., Wang, X., Aguiar, R. L. (2014). Green HetNet CoMP: Energy efficiency analysis and optimization. IEEE Transactions on Vehicular Technology, 64(10), 4670-4683. 15.Busari, S. A., Huq, K. M. S., Felfel, G., Rodriguez, J. (2018, December). Adaptive resource allocation for energy-efficient millimeter-wave massive MIMO networks. In 2018 IEEE Global Communications Conference (GLOBECOM) (pp. 1-6). IEEE.

16.O. E. Ayach, S. Rajagopal, S. Abu-Surra, Z. Pi, and R. W. Heath, "Spatially sparse precoding in millimeter wave MIMO systems," IEEE Transactions on Wireless Communications., vol. 13, no. 3, pp. 1499-1513, Mar. 2014.

17. B. Wang, F. Gao, S. Jin, H. Lin, G. Y. Li, S. Sun, and T. S. Rappaport, "Spatial-wideband effect in massive MIMO with application in mmwave systems," IEEE Communications Magazine., vol. 56, no. 12, pp. 134-141, Dec. 2018.

18. S. Park, A. Alkhateeb, and R. W. Heath, "Dynamic subarrays for hybrid precoding in wideband mmWave MIMO systems", IEEE Transactions on Wireless communications, vol. 16, no. 5, pp. 2907-2920, May 2017.

19. L. Kong, S. Han, and C. Yang, "Hybrid precoding with rate and coverage constraints for wideband massive MIMO systems," IEEE Transactions on Wireless communications, vol. 17, no. 7, pp. 4634- 4647, Jul. 2018.

20. M. Cai, K. Gao, D. Nie, B. Hochwald, J. N. Laneman, H. Huang, and K. Liu, "Effect of wideband beam squint on codebook design in phased-array wireless systems," in Proc. IEEE Global Communication Conferences, Dec. 2016, pp. 1-6.

21. X. Liu and D. Qiao, "Space-time block coding-based beamforming for beam squint compensation," IEEE Wireless Communication Letters, vol. 8, no. 1, pp. 241-244, Feb. 2019.

22. J. Tan and L. Dai, "Delay-Phase Precoding for THz Massive MIMO with Beam Split," 2019 IEEE Global Communications Conference (GLOBECOM), 
Waikoloa, HI, USA, 2019, pp. 1-6, doi: 10.1109/GLOBECOM38437.2019.9014304.

23. Dai, L., Tan, J., Poor, H. V. (2021). Delay-Phase Precoding for Wideband THz Massive MIMO. arXiv preprint arXiv:2102.05211.

24. Sun, S., Rappaport, T. S., Shafi, M., Tang, P., Zhang, J., Smith, P. J. (2018). Propagation models and performance evaluation for 5G millimeterwave bands. IEEE Transactions on Vehicular Technology, 67(9), 8422-8439.

25. Busari, S. A., Huq, K. M. S., Mumtaz, S., Rodriguez, J. (2019, May). Terahertz massive MIMO for beyond-5G wireless communication. In ICC 2019-2019 IEEE International Conference on Communications (ICC) (pp. 16). IEEE.

26. Sun, S., Rappaport, T. S., Thomas, T. A., Ghosh, A., Nguyen, H. C., Kovács, I. Z., ... Partyka, A. (2016). Investigation of prediction accuracy, sensitivity, and parameter stability of large-scale propagation path loss models for $5 \mathrm{G}$ wireless communications. IEEE Transactions on Vehicular Technology, 65(5), 2843-2860.

27. Liebe, H. J., Hufford, G. A., Cotton, M. G. (1993). Propagation modeling of moist air and suspended water/ice particles at frequencies below $1000 \mathrm{GHz}$. 28. Sun, S., MacCartney, G. R., Rappaport, T. S. (2017, May). A novel millimeter-wave channel simulator and applications for 5G wireless communications. In 2017 IEEE International Conference on Communications (ICC) (pp. 1-7). IEEE.

29. Ju, S., Kanhere, O., Xing, Y., Rappaport, T. S. (2019, December). A millimeter-wave channel simulator NYUSIM with spatial consistency and human blockage. In 2019 IEEE Global Communications Conference (GLOBECOM) (pp. 1-6). IEEE.

30. Ju, S., Xing, Y., Kanhere, O., Rappaport, T. S. (2021). Millimeter wave and sub-terahertz spatial statistical channel model for an indoor office building. arXiv preprint arXiv:2103.17127. 